\title{
EFFECT OF PULSE SHAPE AND DURATION ON DYNAMIC RESPONSE OF A FORGING SYSTEM
}

\author{
Arkadiusz TRABKK* \\ *Faculty of Mechanical Engineering and Computer Science, Department of Engineering Fundamentals, \\ University of Bielsko-Biala, ul. Willowa 2, 43-309 Bielsko-Biała, Poland \\ atrabka@ath.bielsko.pl
}

received 26 June 2018, revised 6 December 2019, accepted 10 December 2019

\begin{abstract}
Forging hammers are machines whose operation causes negative effects both at the place of their foundation (the soil settlement) and in their surroundings (e.g., vibrations propagating to the other devices, noise, etc.). Knowledge of the parameters characterizing the time history of the force that arises as a result of impact of a ram on a shaped material is of fundamental importance for the correct analysis of both the structure of the hammer and its impact on the surroundings. In the paper, the effect of the shape and duration of a pulse load on the dynamic response of a hammer-foundation forging system was assessed. An analytical method of description of the forces that arise as a result of impact of the ram on the forged material, using different forms of pulses was presented. The forces defined in this way as loads in a mathematical model of three degrees of freedom forging system were used. The equations of motion derived from d'Alembert's principle were solved numerically in the Matlab program. The analyses for eight forms of the pulse loads with the same pulse sizes but different durations were performed. The results in the graphs were presented. It was found, among other things, that a greater impact on the maximum displacement, velocity and acceleration of each component of the hammer-foundation system as well as on the maximum forces transmitted to the soil has the duration of a pulse than its shape.
\end{abstract}

Key words: Dynamic response, impact load, pulse shape, pulse duration, forging hammer

\section{INTRODUCTION}

Forging hammers are impact action devices. They shape materials using the energy generated before the tool touches them.

There are basically two types of forging hammers: gravity hammers and power hammers (Major, 1980). The first group includes drop hammers and single-acting steam hammers, where during the downstroke, the ram is accelerated by gravity and builds up the impact energy. The second group includes doubleacting hammers, where during the downstroke, in addition to gravity, the ram is accelerated by steam or compressed air. Of these types, the power hammers are most commonly used because they can easily produce high-energy impacts.

The following basic elements can be distinguished in the general diagram of a forging hammer with high-energy impact (Fig. 1): a ram consisting of a rod and a piston 1 , an upper anvil 2 and, if necessary, an upper die 3 attached to it, a frame 4 with guides 5 and the ram drive mechanism 6 , an anvil block 7 with a lower anvil 8 and, if applicable, a lower die 9 attached to it.

Due to the impact nature of the action, high-energy impacts and the need to protect the environment against the negative effects of the forging hammers operation (the soil load, vibrations and noise), spring-damping elements and a foundation are placed under the anvil block. Depending on the type of foundation, the spring-damping elements placed under the anvil block may be pads made of oak beams or a hard felt, or these may be sets of viscous-spring vibro-isolators (Lipiński, 1985; Major, 1980). Nowadays, foundations are most often made either as systems of reinforced concrete structures (Fig. 2a), sometimes replaced with steel supporting structures (Fig. 6a), connected by spring- damping elements, or as reinforced concrete foundation troughs placed directly on the soil (Fig. 2b).

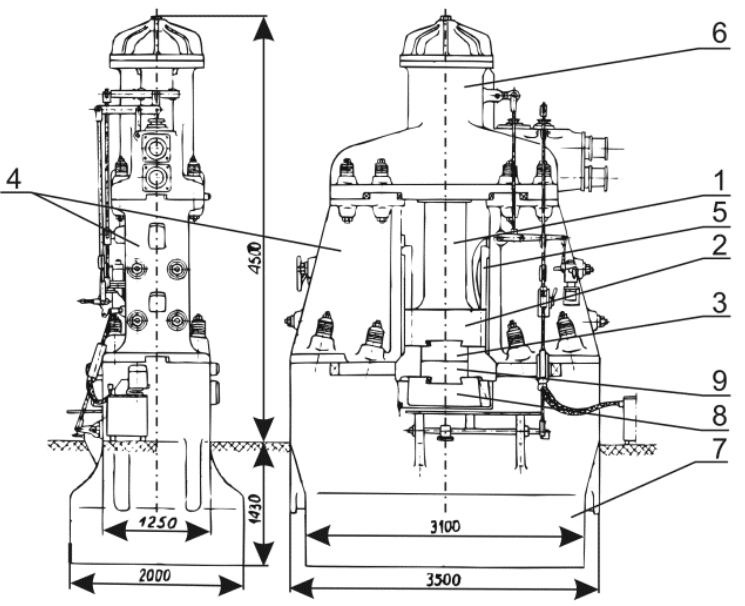

Fig. 1. Structural diagram of a forging hammer with high-energy impact (Majewski and Trąbka, 2006)

The forging process starts with the initiation of the ram movement. The ram hits the forged material at a certain velocity, causing it to deform. At the moment of the impact a part of the kinetic energy, which is accumulated in the ram, changes into the work of plastic deformation of the forged material, and the remaining unused part of the energy gives the ram a return velocity, and above all causes vibrations and elastic deformations of the anvil and foundation. Then the vibrations are transmitted through the foundation to the soil and surroundings. 
The influence of the impact loads on the dynamics of forging devices, as well as the surroundings, for many years has been the subject of discussions, the results of which are presented at scientific conferences, for example, 'International Conference on Structures Under Shock and Impact', are made available as books (Altan et al., 1969; Dresig and Holzweißig, 2010; Gryboś, 1969; Harris ed. and Piersol ed., 2002; Lipiński, 1985; Major, 1980), and above all, are published as articles in scientific journals.

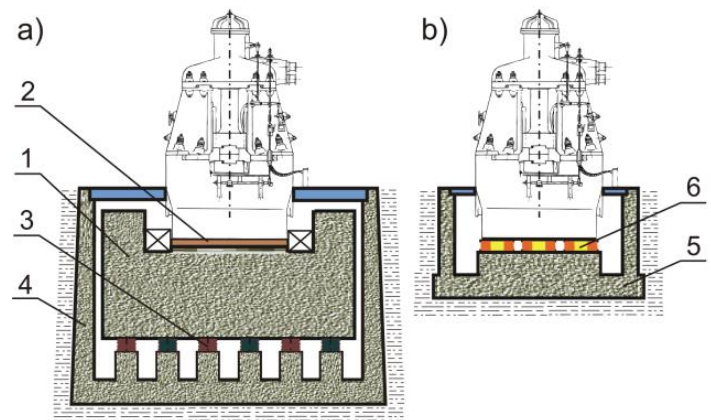

Fig. 2. Examples of foundations for forging hammers: a) a reinforced concrete foundation block 1 with a sub-anvil block pad 2 based on a spring-rubber isolation system 3 in a reinforced concrete foundation trough $4, b$ ) a reinforced concrete foundation trough 5 placed directly on the soil with a set of viscous-spring vibro-isolators 6

Chehab and El Naggar (2003) assumed that the dynamical systems analysed by them set in motion the impact force with a rectangular pulse shape. Using the models with one and two degrees of freedom, they investigated the possibility of reducing the negative impact of the hammers on the surroundings. Leopa (2011) in turn, for a three-mass system, analysed the influence of the impact pulse duration of $0.1 \mathrm{~s}, 0.03 \mathrm{~s}$ and $0.007 \mathrm{~s}$, respectively, on the frequency representation of the considered load. He carried out the analyses for pulse loads of the shapes: haversine and trapezoidal. The influence of the pulse shape generated during an impact on the dynamic response of the forging hammer foundation was investigated in Prolovic et al. (2004). The analyses were carried out for models with one and two degrees of freedom. The pulse loads with rectangular, trapezoidal, parabolic and semisinusoidal shapes were considered. The same pulse duration was assumed $(0.001 \mathrm{~s})$. The study of the dynamic response of a onemass model of a forging hammer both on changes in the shape of the pulse load (rectangular, semi-sinusoidal and symmetric triangular), as well as changes in its duration are presented in Chehab and El Naggar (2004). The same kind of analysis, but used in the study of the dynamics of one and two-mass models of foundations for presses, was presented in Zheng et al. (2014).

Based on the papers found, it can be seen that all processes that occur during forging depend strictly on the load transferred from the ram to the forged material. This load has the form of a pulse and is characterized by its size, shape and duration. The authors of individual papers conduct the dynamic analyses of forging processes either for the selected load durations, or one, or at most several forms of the time history of the forging force. Although in some papers, attempts of the more general analyses are undertaken (Chehab and El Naggar, 2004; Zheng et al., 2014), in relation to the hammer-foundation forging systems, it is carried out using maximally simplified models with one degree of freedom (Chehab and El Naggar, 2004).

Since among the found papers, there was no comprehensive study on the assessment of the effect of the duration and shape of the pulse on the quantities particularly important for assessing the impact of the forging hammer on the surroundings (the maximum displacements, velocities and accelerations of the foundation and the forces transmitted to the soil), in this paper, an analysis was carried out in the above-mentioned scope. The forging system with three degrees of freedom was assessed. The analyses for eight forms of the pulse loads with the same pulse sizes but different durations were performed. The pulse durations were changed from $0.001 \mathrm{~s}$ to $0.2 \mathrm{~s}$ every $0.001 \mathrm{~s}$. The dynamic responses of the forging system for unfavourable forging conditions, that is, cold forging of steel at maximum velocity were determined.

\section{IMPACT LOADS}

The forces that arise during the blows of the ram of the forging hammer against the forged material are the reaction of the system absorbing the impact energy to the impact load (Gryboś, 1969). These forces reach very high values and their durations $\tau$ are very short. They are referred to as the so-called pulse loads, and their size $S(1)$ is determined as equal to the area under the curve representing the time history of the pulse load $P(t)$ (Fig. 3).

$S=\int_{0}^{\tau} P(t) d t$

where: $S$ - the pulse size, $P(t)$ - the time history of the pulse, $\tau$ the pulse duration.

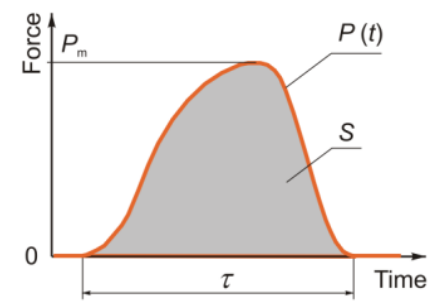

Fig. 3. Pulse load

If the pulse durations $\tau$ are extremely short, that is, satisfy the condition $\tau<0.1 \cdot T_{\min }$ (where: $T_{\min }$ is the smallest period of natural vibration of the system), they are referred to as sudden pulses (Harris ed. and Piersol ed., 2002; Lipiński, 1985). The sudden pulses are fully characterized only by their size $S$. In the description of this type of loads, the shapes of the force time histories are omitted. However, it is assumed that the forces act only at selected points in time (the loads are defined using the Dirac delta function).

If the pulse durations $\tau$ satisfy the condition $0.1 \cdot T_{\min } \leq$ $\tau \leq T_{\max }$ (where: $T_{\max }$ is the largest period of natural vibration of the system), the pulses are called short-term (Lipiński, 1985). In this case, the response of the system is affected by the characteristics of the pulse loads. The short-term pulses are characterized by their shape, defined as the time history of the pulse $P(t)$, size $S$ or amplitude $P_{m}$, as well as duration $\tau$ (Harris ed. and Piersol ed., 2002; Lipiński, 1985; Prolović et al., 2004).

The pulse loads resulting from the blows can take different shapes (Fig. 4). The differences between them result, among others, from the size and shape of the forged material, its temperature and mechanical properties, elastic properties of the materials used to make elements of the forging system, the contact surface geometry and the impact energy (Gryboś, 1969). A large number of factors affecting the time histories of forging forces 
makes their theoretical determination very difficult, therefore, they are determined experimentally (Bieliajew and Popow, 1967).

For theoretical considerations, the real non-linear time histo- ries of the pulses are approximated in various ways, including straight lines or trigonometric functions (Fig. 5). a)

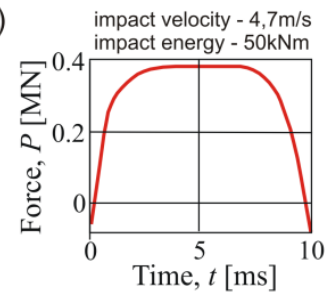

b)

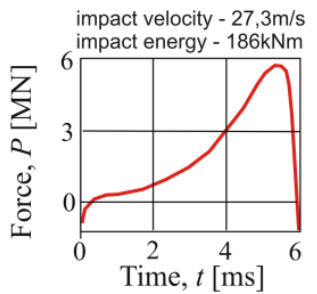

c)

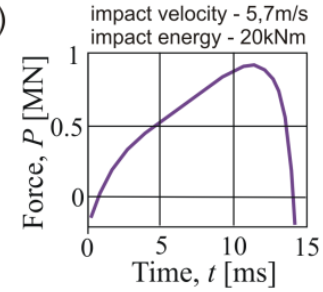

d)

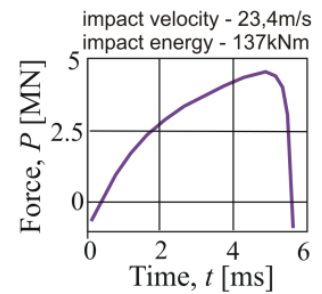

Fig. 4. Time histories of forging forces obtained experimentally by Bieliajew and Popow (1967): a), b) for steel, c), d) for duralumin

a)

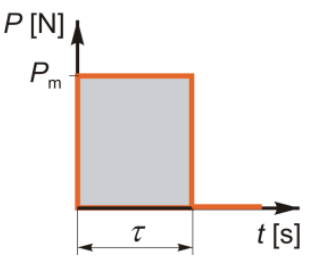

e)

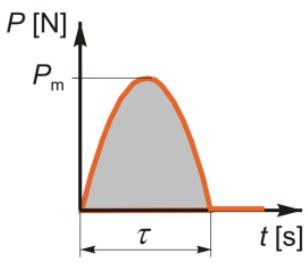

b)

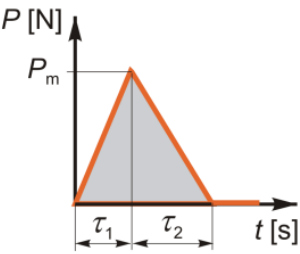

f)

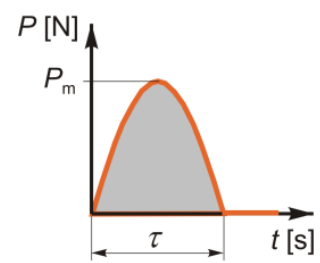

c)

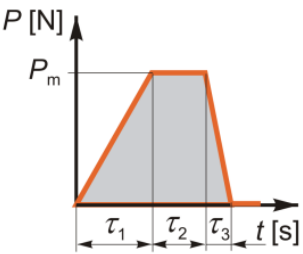

g)

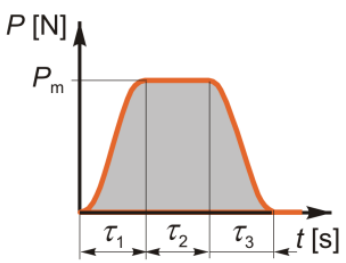

d)

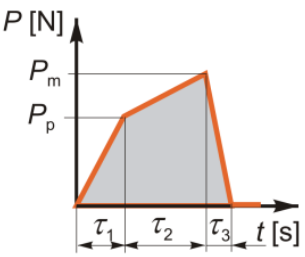

h)

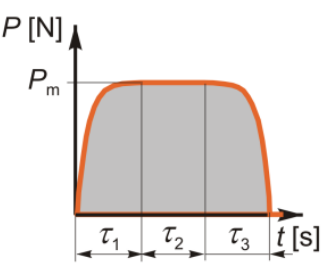

Fig. 5. Shapes of pulse loads: a) rectangular (type A), b) triangular (type B), c) trapezoidal (type C), d) triangular-trapezoidal (type D), e) parabolic (type E), f) semi-sinusoidal (type F), g) versed sine-rectangular (type G), h) exponential-rectangular (type H)

The approximate time histories $P(t)$ of the pulses can be presented in analytical notation as follows:

- $\quad$ Rectangular pulse (Fig. 5a):

$P(t)=\left\{\begin{array}{l}P_{m}=\frac{s}{\tau} \\ 0\end{array}\right.$

- $\quad$ Triangular pulse (Fig. 5b):

$P(t)=\left\{\begin{array}{l}P_{m} \cdot \frac{t}{\tau_{1}}=\frac{2 \cdot S}{\tau_{1}+\tau_{2}} \cdot \frac{t}{\tau_{1}} \\ P_{m} \cdot \frac{\tau_{1}+\tau_{2}-t}{\tau_{2}}=\frac{2 \cdot S}{\tau_{1}+\tau_{2}} \cdot \frac{\tau_{1}+\tau_{2}-t}{\tau_{2}} \\ 0\end{array}\right.$

- $\quad$ Trapezoidal pulse (Fig. 5c):

$P(t)=\left\{\begin{array}{l}P_{m} \cdot \frac{t}{\tau_{1}}=\frac{2 \cdot S}{\tau_{1}+2 \cdot \tau_{2}+\tau_{3}} \cdot \frac{t}{\tau_{1}} \\ P_{m}=\frac{2 \cdot S}{\tau_{1}+2 \cdot \tau_{2}+\tau_{3}} \\ P_{m} \cdot \frac{\tau_{1}+\tau_{2}+\tau_{3}-t}{\tau_{3}}=\frac{2 \cdot S}{\tau_{1}+2 \cdot \tau_{2}+\tau_{3}} \cdot \frac{\tau_{1}+\tau_{2}+\tau_{3}-t}{\tau_{3}} \\ 0\end{array}\right.$

- $\quad$ Triangular-trapezoidal pulse (Fig. 5d):

$P(t)=\left\{\begin{array}{l}u_{P_{m}} \cdot P_{m} \cdot \frac{t}{\tau_{1}} \\ u_{P_{m}} \cdot P_{m}+\left(P_{m}-u_{P_{m}} \cdot P_{m}\right) \cdot \frac{t-\tau_{1}}{\tau_{2}} \\ P_{m} \cdot \frac{\tau_{1}+\tau_{2}+\tau_{3}-t}{\tau_{3}} \\ 0\end{array}\right.$

where: $P_{m}=\frac{2 \cdot S}{u_{P_{m}} \cdot\left(\tau_{1}+\tau_{2}\right)+\tau_{2}+\tau_{3}}$ for

for

for

for

for

for

for

for

for

for

for

for

for

$$
0 \leq t \leq \tau
$$$$
t>\tau
$$

$\tau_{1}<t \leq \tau_{1}+\tau_{2}$

$$
t>\tau_{1}+\tau_{2}
$$

$0 \leq t \leq \tau_{1}$

$\tau_{1}<t \leq \tau_{1}+\tau_{2}$

$\tau_{1}+\tau_{2}<t \leq \tau_{1}+\tau_{2}+\tau_{3}$

$t>\tau_{1}+\tau_{2}+\tau_{3}$ 
- $\quad$ Parabolic pulse (Fig. 5e):

$P(t)= \begin{cases}4 \cdot P_{m} \cdot \frac{t}{\tau} \cdot\left(1-\frac{t}{\tau}\right)=4 \cdot \frac{3}{2} \cdot \frac{s}{\tau} \cdot \frac{t}{\tau} \cdot\left(1-\frac{t}{\tau}\right) & \text { for } \\ 0 & \text { for }\end{cases}$

$0 \leq t \leq \tau$

$t>\tau$

- $\quad$ Semi-sinusoidal pulse (Fig. 5f):

$P(t)=\left\{\begin{array}{l}P_{m} \cdot \sin \left(\pi \cdot \frac{t}{\tau}\right)=\frac{\pi}{2} \cdot \frac{s}{\tau} \cdot \sin \left(\pi \cdot \frac{t}{\tau}\right) \\ 0\end{array}\right.$

for

for

- $\quad$ Versed sine-rectangular pulse (Fig. $5 \mathrm{~g}$ ):

$P(t)=\left\{\begin{array}{l}\frac{P_{m}}{2} \cdot\left(1-\cos \left(\pi \cdot \frac{t}{\tau_{1}}\right)\right) \\ P_{m} \\ \frac{P_{m}}{2} \cdot\left(1+\cos \left(\pi \cdot \frac{t-\tau_{1}-\tau_{2}}{\tau_{3}}\right)\right) \\ 0\end{array}\right.$

for

for

for

for

where: $P_{m}=\frac{2 \cdot S}{\tau_{1}+2 \cdot \tau_{2}+\tau_{3}}$

- Exponential-rectangular pulse (Fig. 5h):

$P(t)=\left\{\begin{array}{l}P_{m} \cdot \frac{1-e^{-2 \cdot \pi \cdot \frac{t}{\tau_{1}}}}{1-e^{-2 \cdot \pi}} \\ P_{m} \\ P_{m} \cdot \frac{1-e^{-2 \cdot \pi \cdot\left(1-\frac{t-\tau_{1}-\tau_{2}}{\tau_{3}}\right)}}{1-e^{-2 \cdot \pi}} \\ 0\end{array}\right.$

for

for

for

for

where: $P_{m}=\frac{S}{\tau_{1} \cdot\left(\frac{1}{1-e^{-2 \cdot \pi}}-\frac{1}{2 \cdot \pi}\right)+\tau_{2}+\tau_{3} \cdot\left(\frac{1}{1-e^{-2 \cdot \pi}-\frac{1}{2 \cdot \pi}}\right)}$

In equations $(2 \div 9)$ : $P(t)$ - the time history of the pulse, $P_{m}-$ the pulse amplitude, $u_{P_{m}}$ - the scaling factor (can take values in the range $0 \div 1$ ), $S$ - the pulse size, $\tau, \tau_{1}, \tau_{2}$ and $\tau_{3}$ - the pulse durations (as indicated in Fig. 5).

The pulse amplitudes $P_{m}$ can be determined based on the size of the pulses $S$ and transformed formulas $(2 \div 9)$ for the areas of the figures representing the approximate time histories of the pulses.

The pulse size $S$ for forging, in the absence of measurement data, according to the information contained in Lipiński (1985) can be estimated based on the empirical relationship (10).

$S=(1+R) \cdot m_{B} \cdot v_{B}=(1+R) \cdot m_{B} \cdot \sqrt{\frac{2 \cdot E_{p}}{m_{B}}}$

where: $m_{B}$ - the mass of the ram with the upper die block, $v_{B}$ the velocity of the ram at the moment of impact, $E_{p}$ - the total energy of the impact of the hammer, $R$ - the coefficient of restitution (the coefficient of impact elasticity). The value of $R$ varies between 0 and 1 , depending upon whether the colliding bodies are of plastic character $(R=0)$, or the impact is completely elastic $(R=1)$ (Gryboś, 1969; Lipiński, 1985; Major, 1980).

\section{CONSIDERED FORGING SYSTEM}

The real forging system (Fig. 6a) containing a steam-air die forging hammer MPM $16000 \mathrm{~B}$ - type 1, and a foundation consisting of a viscous-elastic pad 2, a steel frame 3 , a viscous-spring isolation system 4 and a foundation trough 5 , which rests on an elastic subsoil, was selected as the object of considerations. For the above structure, a physical model with three degrees of freedom was adopted (Fig. 6b).

$$
\begin{array}{r}
0 \leq t \leq \tau_{1} \\
\tau_{1}<t \leq \tau_{1}+\tau_{2} \\
\tau_{1}+\tau_{2}<t \leq \tau_{1}+\tau_{2}+\tau_{3} \\
t>\tau_{1}+\tau_{2}+\tau_{3}
\end{array}
$$

Replacing the real object with the physical model, it was assumed, among other things, that the hammer, the steel frame and the foundation trough are coaxially located, non-deformable material bodies with linear relative motion. The Kelvin-Voigt body, defined by a spring and a dashpot connected in parallel, fulfils the role of constraints between the material bodies. The masses of the material bodies are concentrated in material points, while the elastic and damping constraints are considered as weightless. a)

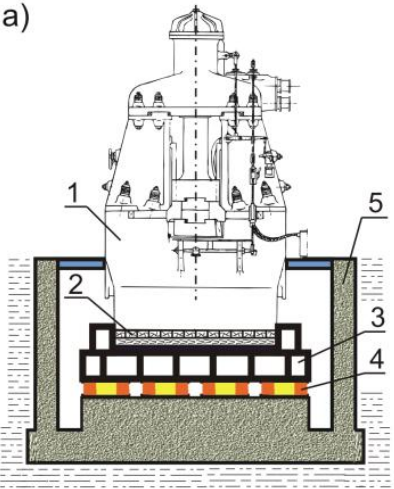

b)

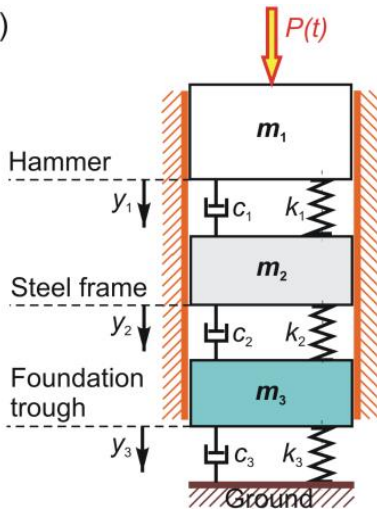

Fig. 6. a) Structural diagram of a real forging system, b) Physical model of the forging system

\section{MATHEMATICAL MODEL AND ITS PARAMETERS}

The mathematical model of the forging system consisting of three mass elements (Fig. 6b) was written in the form of the motion equations (11), which were derived based on the d'Alembert principle for the case of forced-damped vibrations. 
$m_{1} \cdot \ddot{y}_{1}+c_{1} \cdot\left(\dot{y}_{1}-\dot{y}_{2}\right)+k_{1} \cdot\left(y_{1}-y_{2}\right)=P(t)$

$m_{2} \cdot \ddot{y}_{2}+c_{1} \cdot\left(\dot{y}_{2}-\dot{y}_{1}\right)+k_{1} \cdot\left(y_{2}-y_{1}\right)+$

$+c_{2} \cdot\left(\dot{y}_{2}-\dot{y}_{3}\right)+k_{2} \cdot\left(y_{2}-y_{3}\right)=0$

$m_{3} \cdot \ddot{y}_{3}+c_{2} \cdot\left(\dot{y}_{3}-\dot{y}_{2}\right)+k_{2} \cdot\left(y_{3}-y_{2}\right)+$

$+c_{3} \cdot \dot{y}_{3}+k_{3} \cdot y_{3}=0$

where: $m_{1}, m_{2}, m_{3}$ - the masses of the hammer, steel frame and foundation trough, respectively, $c_{1}, c_{2}, c_{3}$ - the damping constants of the viscous-elastic pad, viscous-spring isolation system and soil, respectively, $k_{1}, k_{2}, k_{3}$ - the stiffness of the viscouselastic pad, viscous-spring isolation system and soil, respectively, $P(t)$ - the time history of the pulse, $\ddot{y}_{i}, \dot{y}_{i}, y_{i}$ - the acceleration, velocity and displacement of the $i$-th mass $(i=1,2,3)$, respectively.

The differential equations of motion (11) with initial conditions (12) were solved numerically in the Matlab program.

$t=0 ; \quad y_{1}=y_{2}=y_{3}=0 ; \quad \dot{y}_{1}=\dot{y}_{2}=\dot{y}_{3}=0$

The subsequent time histories of the pulses $P(t)$ described by equations $(2 \div 9)$ as the load were assumed. The integration of the equations was performed using the fourth order Runge-Kutta technique and own calculation scripts.

As the solution of the differential equations the time histories of the displacements, velocities and accelerations of individual elements of the forging system were obtained.

The force transmitted to the soil can be evaluated from equation (13) (Chehab and El Naggar, 2003; Chehab and El Naggar, 2004; Zheng et al., 2014).

$P_{\mathrm{S}}=c_{3} \cdot \dot{y}_{3}+k_{3} \cdot y_{3}$

where: $P_{\mathrm{S}}$ - the force transmitted to the soil, $c_{3}$ - the damping constant of the soil, $k_{3}$ - the stiffness of the soil, $\dot{y}_{3}$ - the velocity of the foundation trough, $y_{3}$ - the displacement of the foundation trough.

The parameters of the numerical model were adopted on the basis of the data characterizing the real foundation of the die forging hammer MPM 16000 B - type, as shown in Fig. 6a. The parameters of the model are summarized in Table 1.

Tab. 1. Parameters of the computational model

\begin{tabular}{|c|c|c|c|c|c|}
\hline Parameter & $\begin{array}{c}\text { Value } \\
{[\mathbf{k g}]}\end{array}$ & Parameter & $\begin{array}{c}\text { Value } \\
{[\mathrm{N} / \mathrm{m}]}\end{array}$ & Parameter & $\begin{array}{c}\text { Value } \\
{[\mathrm{N} \cdot \mathbf{s} / \mathrm{m}]}\end{array}$ \\
\hline$m_{1}$ & 168000 & $k_{1}$ & $950 \cdot 10^{6}$ & $c_{1}$ & $1.84 \cdot 10^{6}$ \\
\hline$m_{2}$ & 18500 & $k_{2}$ & $183 \cdot 10^{6}$ & $c_{2}$ & $3.6 \cdot 10^{6}$ \\
\hline$m_{3}$ & 248200 & $k_{3}$ & $8329 \cdot 10^{6}$ & $c_{3}$ & $12.26 \cdot 10^{6}$ \\
\hline
\end{tabular}

\section{ANALYSIS PARAMETERS, RESULTS AND DISCUSSION}

To investigate the effect of the shape and duration of the pulse load on the dynamic response of the hammer-foundation forging system and the forces transmitted to the surroundings, comprehensive analyses were performed.

A single hit of the ram was analysed. The analyses involved eight forms of the pulse loads (Fig. 5) with the same pulse sizes $S=79793 \mathrm{~N} \cdot \mathrm{s}$ but different durations.
The pulse size was determined on the basis of equation (10) (Lipiński, 1985) assuming that the impact of the ram and the upper die block with the total mass $m_{B}=8085 \mathrm{~kg}$ occurs with the maximum kinetic energy, which for the considered die hammer is $E_{p}=175000 \mathrm{~J}$, and the coefficient of restitution $R$ has the value, which according to Dresig and Holzweißig (2010), Lipiński (1985) and Major (1980), corresponds to performing heavy works on the die hammers, that is, cold forging of steel $(R=0.5)$.

The pulse durations $\tau$ were changed from $0.001 \mathrm{~s}$ to $0.2 \mathrm{~s}$ every $0.001 \mathrm{~s}$. The lower limit value was adopted based on the literature data (Altan et al., 1969; Leopa, 2011), while the upper limit was calculated based on the condition of occurrence of the short-term pulses (see chapter 2). For the triangular pulse, it was adopted that $\tau_{1}=\tau_{2}$, while for the pulses: trapezoidal, triangulartrapezoidal, versed sine-rectangular as well as exponentialrectangular, it was assumed that $\tau_{1}=\tau_{3}=0.2 \cdot \tau$. For the triangular-trapezoidal pulse, it was assumed, furthermore, that $u_{P_{m}}=0.7$.

On the basis of the results, the pulse amplitudes, the maximum displacements, velocities and accelerations of the individual elements of the computational model as well as the maximum forces transmitted to the soil were determined.

The pulse amplitudes are shown in Fig. 7 as a function of the shape and duration of the pulses.

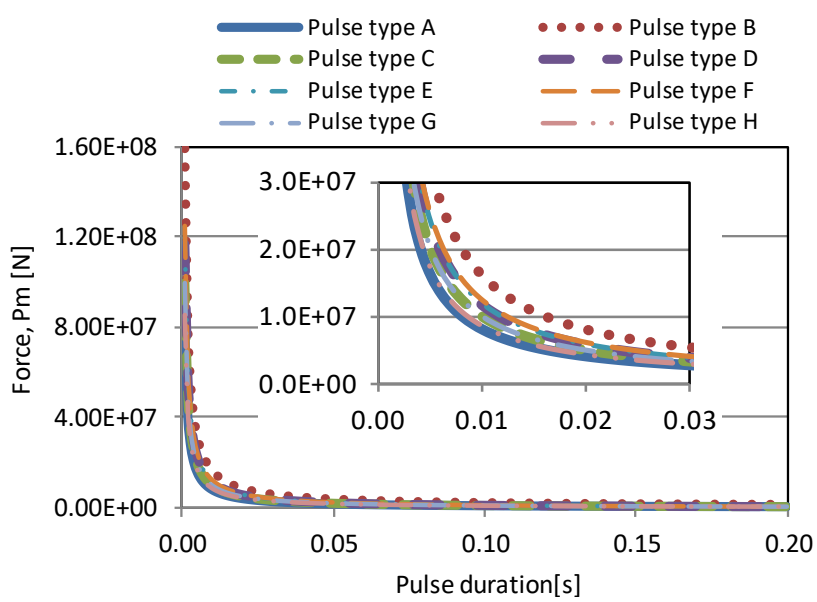

Fig. 7. The pulse amplitudes as function of shape and duration of pulses

Fig. 7 shows that for the same pulse sizes, regardless of their duration, the force reaches the highest value for the triangular pulse, while the maximum forces corresponding to the remaining pulses are lower by $21 \%$ for the semi-sinusoidal pulse, by $26 \%$ for the triangular-trapezoidal and parabolic pulse, by $37 \%$ for the versed sine-rectangular and trapezoidal pulse, by $50 \%$ for rectangular and exponential-rectangular pulse, respectively.

The maximum displacements, velocities and accelerations of the hammer and foundation trough in dependence of the shape and duration of the pulses are shown in Figs. 8-10.

The displacements, velocities and accelerations of the hammer and foundation trough reach the highest values for pulses with the shortest duration (Figs. 8-10). When the duration of the pulses increases, these values decrease. At the same time, depending on the pulse shape, the differences between the maximum displacements of the individual elements increase. 
a)

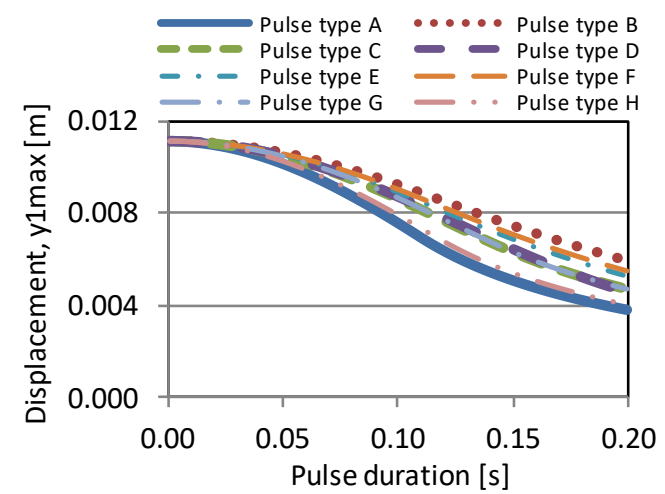

b)

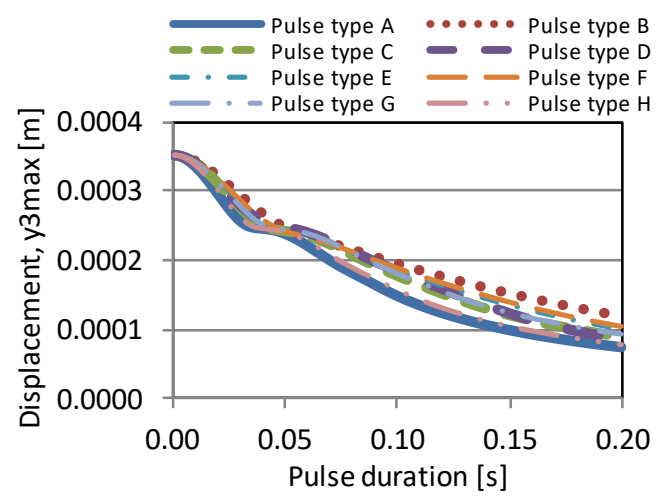

Fig. 8. Effect of shape and duration of pulses on the maximum displacement: a) of the hammer, b) of the foundation trough

a)

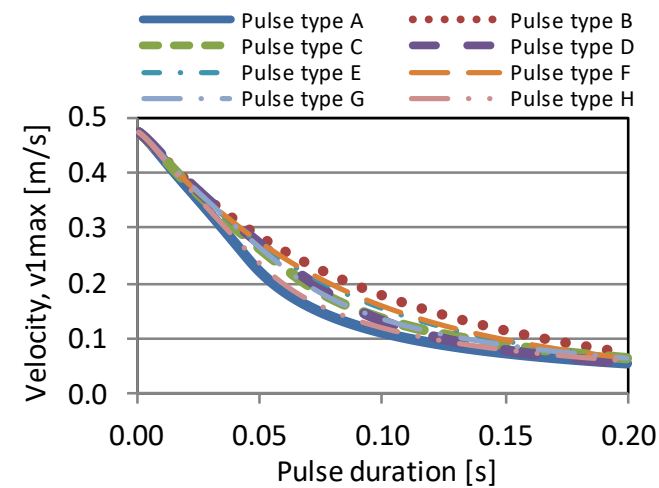

b)

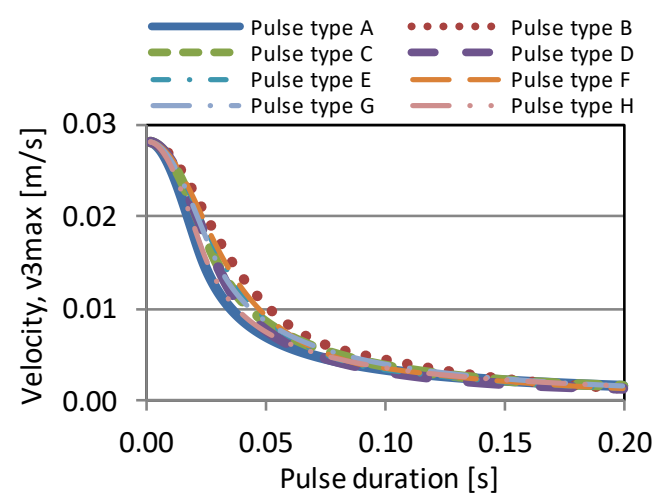

Fig. 9. Effect of shape and duration of pulses on the maximum velocity: a) of the hammer, b) of the foundation trough

a)

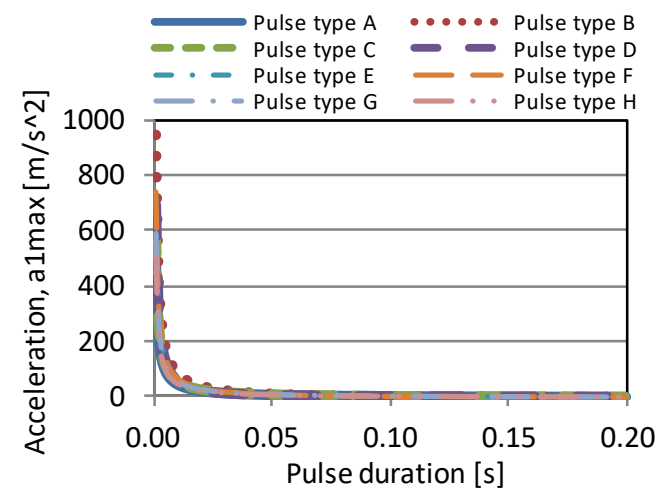

b)

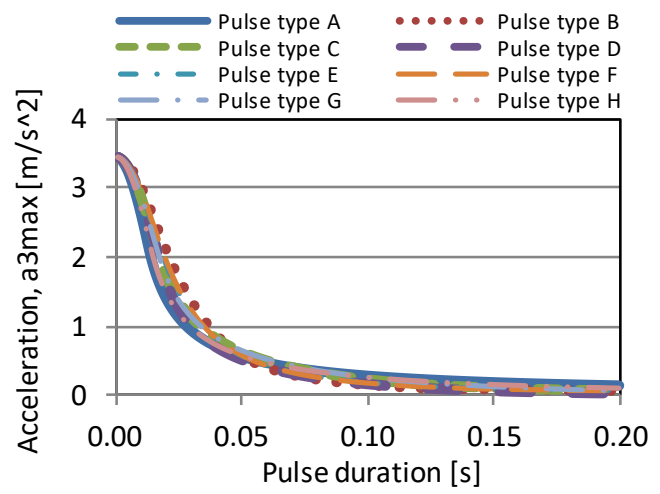

Fig. 10. Effect of shape and duration of pulses on the maximum acceleration: a) of the hammer, b) of the foundation trough

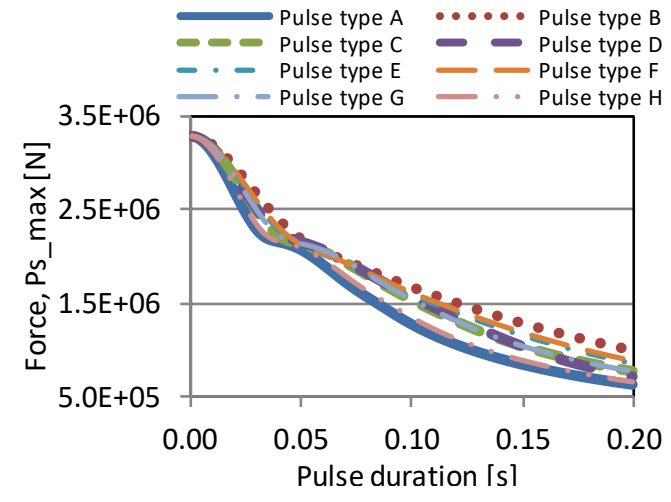

Fig. 11. The maximum forces transmitted to the soil as a function of shape and duration of pulses
Similar observations can be made regarding the maximum forces transmitted to the soil from Fig. 11.

\section{SUMMARY AND CONCLUSIONS}

In the paper, the effect of the duration and shape of a pulse load on the dynamic response of a hammer-foundation forging system was assessed. The forging system with three degrees of freedom was analysed. The analyses for eight forms of the pulse loads with the same pulse sizes but different durations were performed. The parameters of the analysis for unfavourable forging conditions, that is, cold forging of steel at maximum velocity were determined. 
To investigate the effect of the pulse shape and duration on the dynamic response of the hammer-foundation forging system, the maximum displacements, velocities and accelerations of the hammer and foundation as well as the maximum forces transmitted to the soil were determined.

The results of the numerical analyses as a set of graphs were presented. Based on the results of the analyses, it was found that:

- A greater impact on the dynamic response of a forging system has the duration of a pulse than its shape;

- The displacements, velocities and accelerations of the forging system components as well as the forces transmitted to the soil reach the highest values for the pulses with the shortest duration;

- If the pulse duration is less than $0.01 \mathrm{~s}$, the system response is insensitive to the shape of the pulse;

- As the pulse duration increases, the response of the forging system decreases.

Since, as the results of the analyses show, the shape of a pulse has a little effect on the dynamic response of the forging system, during the design work or checking the influence of the structure with known parameters on the soil and surroundings, theoretically, pulse loads of any shape can be used in calculation models. However, since with prolonging the pulse duration, the dynamic responses reach the highest values for the triangular pulse, it seems expedient to use this shape in the calculations.

Due to the large impact of the pulse duration on the maximum displacements and velocities of the forging system components as well as the forces transmitted to the soil, it is important that the pulse duration corresponds to the real forging conditions. Therefore, the analyses should be performed for the experimentally confirmed durations of the contact between the interacting materials.

If the results of the analyses show that for the adopted model parameters of the forging system, the permissible values of the foundation displacements (according to Lipiński (1985) and Major (1980)) or the permissible soil stress (according to Lipiński (1985)) have been exceeded and it is not possible to change these parameters, the only way to limit the negative impact of the forging hammer on the soil and surroundings will be such a modification of the parameters of the forming process, that will prolong the duration of contact between the interacting materials. The same actions should be taken to ensure the proper working conditions for hammerman when the permissible levels of the hammer vibration amplitudes are exceeded (according to Lipiński (1985) and Major (1980)).

\section{REFERENCES}

1. Altan T., Henning H.J., Sabroff A.M. (1969), Sixth interim topical report on a study of the mechanics of closed-die forging, Battelle Memorial Institute, Columbus Laboratories, Ohio.

2. Bieliajew J., Popow A. (1967), Experimental study of the loads of colliding parts of hammers during impact, Forging and Stamping Production (in Russian), 2, 26-30.

3. Chehab A.G., El Naggar M.H. (2003), Design of efficient base isolation for hammers and presses, Soil Dynamics and Earthquake Engineering, 23 (2), 127-141.

4. Chehab A.G., El Naggar M.H. (2004), Response of block foundations to impact loads, Journal of Sound and Vibration, 276(1-2), 293-310.

5. Dresig H., Holzweißig F. (2010), Dynamics of machinery, SpringerVerlag, Berlin.

6. Gryboś R. (1969), Theory of impact in discrete mechanical systems (in Polish), PWN.

7. Harris C.M. ed., Piersol A.G. ed. (2002), Harris' Shock and Vibration Handbook, McGraw-Hill, New York.

8. Leopa A. (2011), Parametric method for dynamic analysis of mechanical impulsive systems actions, Proc. of The Romanian Academy, Series A, 12 (1), 63-70.

9. Lipiński J. (1985), Machine foundations (in Polish). Wyd. Arkady, Warszawa.

10. Majewski L., Trąbka A. (2006), Assessment of dynamical properties of steam-air die hammer MPM 10000 B, TRANSFER 2006: The utilization of the new knowledge in the engineering practice, VIII International Scientific Conference, Trencin, 28-29 september, 2 dil.

11. Major A. (1980), Dynamics in civil engineering - analysis and design - volume II, Budapest, Akademiai Kiado.

12. Prolović V., Bonić Z., Živković S. (2004), Dependence of the basic dynamic parameters on the disturbing force of the impact impulse type with different forms, Facta Universitatis, Series: Architecture and Civil Engineering, 3 (1), 23-31.

13. Zheng E., Zhou X., Zhu S. (2014), Dynamic response analysis of block foundations with nonlinear dry friction mounting system to impact loads, Journal of Mechanical Science and Technology, 28 (7), 2535-2548. 